\title{
Existence of the Optimal Control for Stochastic Boundary Control Problems Governed by Semilinear Parabolic Equations
}

\author{
Weifeng Wang and Baobin Wang \\ School of Mathematics and Statistics, South-Central University for Nationalities, Wuhan, Hubei 430074, China \\ Correspondence should be addressed to Weifeng Wang; wwf87487643@163.com
}

Received 2 July 2014; Accepted 21 August 2014; Published 28 September 2014

Academic Editor: Quanxin Zhu

Copyright (C) $2014 \mathrm{~W}$. Wang and B. Wang. This is an open access article distributed under the Creative Commons Attribution License, which permits unrestricted use, distribution, and reproduction in any medium, provided the original work is properly cited.

We study an optimal control problem governed by a semilinear parabolic equation, whose control variable is contained only in the boundary condition. An existence theorem for the optimal control is obtained.

\section{Introduction}

Control theory is a mathematical description of how to act optimally to gain future rewards. Since the necessary conditions of optimal problems were established for the deterministic control systems by Pontryagin's group [1] in the 1950s and 1960s, a lot of work has been done not only on the deterministic case but also on the stochastic case. To understand the deterministic case of optimal control problems governed by partial differential equations, we can see the classical book written by Lions [2] in 1971. And to the stochastic case, we can see [3-8] and so forth. The boundary control problems of stochastic partial differential equations have been developing fast and very active in recent years, including the boundary exact controllability $[9,10]$ and the maximum principle for boundary control [11].

In our paper, this kind of boundary control problem is extensively studied in many papers in the case of deterministic control systems. For example, we can see Chai [12]. But in the stochastic case, as we known, there are only a few results for this problem. One of the difficulties is that the properties of the solution of the state equation are not clear compared with the deterministic case.

In many papers, the authors only assume that the optimal controls exist for their problems, and they have not proved the existence. In our life, we always only want to use the necessary conditions to find the optimal control and achieve our goal, but the existence for the control problems is also important. Some useful results have been obtained, for instance, in
[13-16]. About the control problems governed by semilinear parabolic equations, we can see $[17,18]$. In this paper, the necessary conditions for our problem have been worked out by a member of our team in the paper [19]. So, in this paper, we only consider the existence of the optimal control as a complement for [19].

The rest of this paper is organized as follows. Section 2 begins with a general formulation of our stochastic optimal control problem. In Section 3, we give two important lemmas of our problem. And we give the existence result of the optimal control in Section 4.

\section{Preliminaries}

Let $(\Omega, \mathfrak{F}, P)$ be a complete probability space, equipped with a right-continuous filtration $\left\{\mathfrak{F}_{t}\right\}_{t \geq 0}$, on which a $d$-dimensional mutually independent standard Brownian motion $\{w(t)\}_{t \geq 0}=$ $\left\{\left(w_{1}(t), \ldots, w_{d}(t)\right)\right\}_{t \geq 0}$ is defined. Moreover, we assume that $\mathfrak{F}_{t}$ is the augmentation of $\sigma\{w(s) \mid 0 \leq s \leq t\}$ by all the $P$-null sets of $\mathfrak{F}$. For a fixed $0<T<+\infty$, we denote the $\sigma$-algebra of predictable sets on $\Omega \times[0, T]$ by $\mathfrak{P}$. Let $O$ be an open bounded subset in $R^{n}$ with a smooth boundary $\Gamma$. Let $Q=O \times(0, T)$ and $\Sigma=\Gamma \times(0, T)$, and $\mathfrak{B}(\mathfrak{X})$ stands for the $\sigma$-algebra of all Borel subsets of $\mathfrak{X}$, where $\mathfrak{X}$ is a topological space. $L^{2}(\cdot)$ denotes the corresponding square-integral space, and $H^{1}(\cdot)$ denotes the Sobolev space $W^{1,2}(\cdot)$. The positive constants $M$ and $C$ can be different in different place throughout this paper. 
Let $U$ be the set of $u(x, t)$ satisfying the following:

(1) $u(x, t): \Omega \times \Gamma \times(0, T) \rightarrow R$ is $\mathfrak{B}\left(R^{n-1}\right) \times \mathfrak{B}([0, T])-$ measurable and $\mathfrak{F}_{t}$-adapted;

(2) $E \int_{\Sigma}|u(x, t)|^{2} d \rho(x) d t<+\infty$, where $\rho(x)$ denotes the usual $(n-1)$-dimensional Lebesgue measure on $\Gamma$.

The set of admissible controls $U_{\mathrm{ad}}$ is a convex, closed subset of $U$.

Now we consider the following stochastic distributed control system with $R$-value control processes in $\Omega \times \Sigma$ :

$$
\begin{gathered}
d y(x, t)=[A y(x, t)+f(x, t, y(x, t))] d t \\
+[B y(x, t)+g(x, t, y(x, t))] d w(t) \quad \text { in } Q, \\
\partial_{v_{A}} y(x, t)=h(x, t, y(x, t), u(x, t)) \text { on } \Sigma, \\
y(x, 0)=y_{0}(x) \quad \text { in } O,
\end{gathered}
$$

where $y_{0} \in L^{2}(O)(P$-a.s. $)$ is $\mathfrak{F}_{0}$-adapted, and the differential operators $A, B$ are defined as follows:

$$
\begin{gathered}
A y=\sum_{i, j=1}^{n} \partial_{x_{j}}\left[a^{i j} \partial_{x_{i}} y+b^{j} y\right]+\sum_{j=1}^{n} d^{j} \partial_{x_{j}} y+c y, \\
B^{k} y=\sum_{i=1}^{n} \sigma^{i k} \partial_{x_{i}} y+\eta^{k} y, \quad k=1, \ldots, d, \\
\partial_{v_{A}} y=\sum_{j=1}^{n}\left\{\sum_{i=1}^{n}\left[\partial_{x_{i}} y\right]+b^{j} y\right\} v_{j},
\end{gathered}
$$

where $v(x)$ denotes the outward unit normal vector to $\Gamma$ at the point $x$. We can see [12] for more details to the Neumann boundary condition in the trace sense. $f, h$ are $R$ value functions and $g$ is a $R^{d}$-value function.

The cost functional is defined as follows:

$$
\begin{aligned}
J\left(y^{u}(\cdot, \cdot), u(\cdot, \cdot)\right)= & E \int_{Q} L\left(x, t, y^{u}(x, t)\right) d x d t \\
& +E \int_{\Sigma} l\left(x, t, y^{u}(x, t), u(x, t)\right) d \rho(x) d t \\
& +E \int_{O} r\left(x, y^{u}(x, T)\right) d x
\end{aligned}
$$

where $y^{u}(x, t)$ denotes the adapted solution of the state equation (1) corresponding to $u(x, t)$ and $L, l, r$ are $R$-value functions.

From the above content, our optimal control problem can be stated as follows.

Problem $(P)$. Find a $\bar{u}(\cdot, \cdot) \in U_{\text {ad }}$ such that

$$
J\left(y^{\bar{u}}(\cdot, \cdot), \bar{u}(\cdot, \cdot)\right)=\inf _{u(\cdot,) \in U_{\mathrm{ad}}} J\left(y^{u}(\cdot, \cdot), u(\cdot, \cdot)\right) .
$$

Any $\bar{u}(\cdot, \cdot) \in U_{\text {ad }}$ satisfying the above identity is called an optimal control, and the corresponding state $y^{\bar{u}}(\cdot, \cdot)$ is called an optimal trajectory. $\left(y^{\bar{u}}(\cdot, \cdot), \bar{u}(\cdot, \cdot)\right)$ is called an optimal pair.

We assume that the following conditions hold.

$\left(H_{1}\right)$ The coefficients $a=\left(a^{i j}\right), b=\left(b^{j}\right), c, d=\left(d^{j}\right)$, $\sigma=\left(\sigma^{i j}\right)$, and $\eta=\left(\eta^{k}\right)$ are measurable in $(x, t)$ with values in the sets of real symmetrical $n \times n$ matrices, $R^{n}, R, R^{n}, R^{n \times d}$, and $R^{d}$, respectively. The real function $y_{0}(x)$ is $\mathfrak{F}_{0} \times \mathfrak{B}\left(R^{n}\right)$ measurable and $E\left\|y_{0}(x)\right\|_{L^{2}(O)}^{2} \leq K$, where $K$ is a positive constant. And the functions $a=\left(a^{i j}\right), b=\left(b^{j}\right), c, d=\left(d^{j}\right)$, $\sigma=\left(\sigma^{i j}\right)$, and $\eta=\left(\eta^{k}\right)$ are all bounded by $K$ in absolute value. Furthermore, the matrix $S=\left(a^{i j}-(1 / 2) \sum_{k=1}^{d} \sigma^{i k} \sigma^{j k}\right)$ is uniformly positive definite, which means

$$
\xi^{\top} S \xi \geq \lambda|\xi|^{2}, \quad \forall(x, t) \in Q, \forall \xi \in R^{n}
$$

for a constant $\lambda>0$.

$\left(H_{2}\right)$ Consider $f: \Omega \times R^{n} \times[0, T] \times R \rightarrow R, g: \Omega \times R^{n} \times$ $[0, T] \times R \rightarrow R^{d}$, and $h: \Omega \times R^{n-1} \times[0, T] \times R \times R \rightarrow R$ to be functions which satisfy the following properties:

(i) $f$ is $\mathfrak{P} \times \mathfrak{B}\left(R^{n}\right) \times \mathfrak{B}(R) / \mathfrak{B}(R)$-measurable, $g$ is $\mathfrak{P} \times$ $\mathfrak{B}\left(R^{n}\right) \times \mathfrak{B}(R) / \mathfrak{B}\left(R^{d}\right)$-measurable, and $h$ is $\mathfrak{P} \times$ $\mathfrak{B}\left(R^{n-1}\right) \times \mathfrak{B}(R) \times \mathfrak{B}(R) / \mathfrak{B}(R)$-measurable;

(ii) $f(\cdot, \cdot, 0), g_{k}(\cdot, \cdot, 0) \in L^{2}(Q)$ and $E \int_{0}^{T} \int_{\Gamma} \mid h(x, t, 0, u(x$, t)) $\left.\right|^{2} d \rho(x) d t<+\infty(k=1, \ldots, d, \forall u \in U) ;$

(iii) there exists a positive constant $M>0$ such that, for $\forall y_{1}, y_{2} \in R$, we have

$$
\begin{aligned}
& \left|f\left(x, t, y_{1}\right)-f\left(x, t, y_{2}\right)\right| \\
& \quad+\sum_{k=1}^{d}\left|g_{k}\left(x, t, y_{1}\right)-g_{k}\left(x, t, y_{2}\right)\right| \leq M\left|y_{1}-y_{2}\right|
\end{aligned}
$$

uniformly in $(\omega, x, t) \in \Omega \times Q$ and

$$
\left|\frac{\partial h}{\partial y}(x, t, y, u)\right|+\left|\frac{\partial h}{\partial u}(x, t, y, u)\right| \leq M
$$

uniformly in $(\omega, x, t, u) \in \Omega \times Q \times R$ and

$$
\frac{\partial h}{\partial y}(x, t, y, u) \leq 0
$$

for almost every $(\omega, x, t, u) \in \Omega \times Q \times R$.

$\left(H_{3}\right)$ Consider $L: R^{n} \times[0, T] \times R \rightarrow R, l: R^{n-1} \times[0, T] \times$ $R \times R \rightarrow R$, and $r: R^{n} \times R \rightarrow R$ to be maps such that the following conditions are satisfied:

(i) $L$ is $\mathfrak{B}\left(R^{n}\right) \times \mathfrak{B}([0, T]) \times \mathfrak{B}(R) / \mathfrak{B}(R)$-measurable, $l$ is $\mathfrak{B}\left(R^{n-1}\right) \times \mathfrak{B}([0, T]) \times \mathfrak{B}(R) \times \mathfrak{B}(R) / \mathfrak{B}(R)$-measurable, and $r$ is $\mathfrak{B}\left(R^{n}\right) \times \mathfrak{B}(R) / \mathfrak{B}(R)$-measurable;

(ii) $L(\cdot, \cdot, 0) \in L^{2}(Q)$ and $l(\cdot, \cdot, 0, u(\cdot, \cdot)) \in L^{2}(\Sigma)$ for every $u(\cdot, \cdot) \in U$ and $r(\cdot, 0) \in L^{2}(O)$;

(iii) $L, r$ are continuous and continuously differentiable functions with respect to the state $y(x, t)$ and $l$ is a 
continuous and continuously differentiable function with respect to the state $y(x, t)$ and the control variable $u(x, t)$; moreover, there exists a constant $M>$ 0 such that

$$
\begin{gathered}
\left|\frac{\partial L}{\partial y}(x, t, y)\right|+\left|\frac{\partial r}{\partial y}(x, y)\right| \leq M \quad \text { uniformly in }(x, t) \in Q \\
\left|\frac{\partial l}{\partial y}(x, t, y, u)\right|+\left|\frac{\partial l}{\partial u}(x, t, y, u)\right| \leq M \\
\text { uniformly in }(x, t, u) \in Q \times R .
\end{gathered}
$$

\section{Some Basic Lemmas}

First, we give the definition of solution of the state equation (1).

Definition 1. We say a function $y(x, t) \in L_{\mathfrak{F}}^{2}(\Omega \times(0, T)$; $\left.H^{1}(O)\right)$ is a weak solution of the state equation (1), if it satisfies

$$
\begin{aligned}
\int_{O} y(t) \phi d x \\
=\int_{O} y_{0} \phi d x-\sum_{i, j=1}^{n} \int_{0}^{t} \int_{O} a^{i j} \partial_{x_{i}} y \partial_{x_{j}} \phi d x d s \\
\quad-\sum_{j=1}^{n} \int_{0}^{t} \int_{O} b^{j} y \partial_{x_{j}} \phi d x d s+\sum_{j=1}^{n} \int_{0}^{t} \int_{O} d^{j} \partial_{x_{j}} y \phi d x d s \\
\quad+\int_{0}^{t} \int_{O} c y \phi d x d s+\int_{0}^{t} \int_{O} f(x, s, y) \phi d x d s \\
\quad \times \int_{0}^{t} \int_{\Gamma} h(x, s, y) \phi d \rho(x) d s \\
\quad+\sum_{i=1}^{n} \int_{0}^{t} \int_{O} \sigma^{i} \partial_{x_{i}} y \phi d x d w(s) \times \int_{0}^{t} \int_{O} \eta y \phi d x d w(s) \\
\quad+\int_{0}^{t} \int_{O} g(x, s, y) \phi d x d w(s), \quad P-\text { a.s. }
\end{aligned}
$$

for every $\phi \in H^{1}(O)$ and almost every $t \in(0, T]$.

Lemma 2 (Yu and Liu [19]). Let the conditions $\left(H_{1}\right)$ and $\left(H_{2}\right)$ hold; then the state equation (1) has a unique (weak) solution $y(x, t) \in L_{\widetilde{F}}^{2}\left(\Omega \times(0, T) ; H^{1}(O)\right)$ for every control $u(x, t) \in U$. Moreover, there exists a constant $C>0$ such that

$$
\sup _{0 \leq t \leq T} E\|y(t)\|_{L^{2}(O)}^{2}+\|y\|_{L_{\mathfrak{F}}^{2}\left(\Omega \times(0, T) ; H^{1}(O)\right)}^{2} \leq C .
$$

Remark 3. We can see that, by Lemma 2, the cost functional $J\left(y^{u}(\cdot, \cdot), u(\cdot, \cdot)\right)$ can be denoted by $J(u(\cdot, \cdot))$ for simplicity.
Lemma 4. Let one designate by $y_{m}(x, t)$ the solution of the state equation (1) corresponding to $u_{m}(x, t) \in U_{a d}$ and suppose that $u_{m}(x, t) \rightarrow \bar{u}(x, t)$ weakly in $L^{2}(\Sigma)$. Then, for any fixed $\omega \in \Omega$, one has

$$
\begin{array}{r}
y_{m}(x, t) \longrightarrow y_{\bar{u}}(x, t) \quad \text { in } L^{2}\left((0, T) ; L^{2}(O)\right) \\
\text { as } m \longrightarrow+\infty .
\end{array}
$$

Proof. From the state equation (1), we have

$$
\begin{gathered}
y_{m}(x, t)-y_{\bar{u}}(x, t) \\
=\int_{0}^{t}\left[A\left(y_{m}(x, s)-y_{\bar{u}}(x, s)\right)+f\left(x, s, y_{m}(x, s)\right)\right. \\
\left.\quad-f\left(x, s, y_{\bar{u}}(x, s)\right)\right] d s \\
+\int_{0}^{t}\left[B\left(y_{m}(x, s)-y_{\bar{u}}(x, s)\right)+g\left(x, s, y_{m}(x, s)\right)\right. \\
\left.\quad-g\left(x, s, y_{\bar{u}}(x, s)\right)\right] d w(s) .
\end{gathered}
$$

By the definition of the operators $A, B$, we apply Itô's formula to $\left(y_{m}(x, t)-y_{\bar{u}}(x, t)\right)^{2}$, and then we can get

$$
\begin{aligned}
& E \int_{O}\left|y_{m}(x, t)-y_{\bar{u}}(x, t)\right|^{2} d x \\
& \triangleq E \int_{O}|\zeta(x, t)|^{2} d x \\
& =2 E \int_{0}^{t} \int_{\Gamma}\left[h\left(x, s, y_{m}(x, s), u_{m}(x, s)\right)\right. \\
& \left.-h\left(x, s, y_{\bar{u}}(x, s), \bar{u}(x, s)\right)\right] \zeta(x, s) d \rho(x) d s \\
& -2 E \sum_{i, j=1}^{n} \int_{0}^{t} \int_{O} a^{i j} \partial_{x_{i}} \zeta(x, s) \partial_{x_{j}} \zeta(x, s) d x d s \\
& +2 E \sum_{j=1}^{n} \int_{0}^{t} \int_{O}\left[d^{j}-b^{j}\right] \partial_{x_{j}} \zeta(x, s) \zeta(x, s) d x d s \\
& +2 E \int_{0}^{t} \int_{O}\left\{c \zeta^{2}(x, s)\right. \\
& +\left[f\left(x, s, y_{m}(x, s)\right)-f\left(x, s, y_{\bar{u}}(x, s)\right)\right] \\
& \times \zeta(x, s)\} d x d s \\
& +E \sum_{i, j=1}^{n} \int_{0}^{t} \int_{O} \sigma^{i} \sigma^{j} \partial_{x_{i}} \zeta(x, s) \partial_{x_{j}} \zeta(x, s) d x d s \\
& +E \int_{0}^{t} \int_{O}|\eta \zeta(x, s)|^{2} \\
& +\left(g\left(x, s, y_{m}(x, s)\right)-g\left(x, s, y_{\bar{u}}(x, s)\right)\right)^{2} d x d s
\end{aligned}
$$




$$
\begin{aligned}
& +2 E \sum_{i=1}^{n} \int_{0}^{t} \int_{O} \sigma^{i} \eta \partial_{x_{i}} \zeta(x, s) \zeta(x, s) d x d s \\
& +2 E \sum_{i=1}^{n} \int_{0}^{t} \int_{O} \sigma^{i} \eta \partial_{x_{i}} \zeta(x, s) \\
& \times\left(g\left(x, s, y_{m}(x, s)\right)\right. \\
& +2 E \int_{0}^{t} \int_{O}^{\eta \zeta}(x, s) \\
& \times\left(g\left(x, s, y_{m}(x, s)\right)-g\left(x, s, y_{\bar{u}}(x, s)\right)\right) d x d s
\end{aligned}
$$

By $\left(H_{2}\right)$, it follows that

$$
\begin{gathered}
\int_{0}^{t} \int_{\Gamma}\left[h\left(x, s, y_{m}(x, s), u_{m}(x, s)\right)\right. \\
\left.-h\left(x, s, y_{\bar{u}}(x, s), \bar{u}(x, s)\right)\right] \zeta(x, s) d \rho(x) d s \\
\leq M \int_{0}^{t} \int_{\Gamma}\left[\left|u_{m}(x, s)-\bar{u}(x, s)\right|+|\zeta(x, s)|\right] \\
\times \zeta(x, s) d \rho(x) d s .
\end{gathered}
$$

For any fixed $\omega \in \Omega$,

$$
\begin{aligned}
& \int_{0}^{t} \int_{\Gamma}\left|u_{m}(x, s)-\bar{u}(x, s)\right| \\
& \times\left|y_{m}(x, s)-y_{\bar{u}}(x, s)\right| d \rho(x) d s \\
& \leq C\left\|u_{m}(x, s)-\bar{u}(x, s)\right\|_{H^{-1}(\Sigma)} \\
& \quad \times\left\|y_{m}(x, s)-y_{\bar{u}}(x, s)\right\|_{H^{1}(\Sigma)} .
\end{aligned}
$$

Thanks to Lemma 2 and because the imbedding

$$
L^{2}(\Sigma) \hookrightarrow H^{-1}(\Sigma)
$$

is compact, we can obtain

$$
\begin{aligned}
& E \int_{0}^{t} \int_{\Gamma}\left|u_{m}(x, s)-\bar{u}(x, s)\right| \\
& \times\left|y_{m}(x, s)-y_{\bar{u}}(x, s)\right| d \rho(x) d s \longrightarrow 0 \\
& \text { as } m \longrightarrow+\infty
\end{aligned}
$$

From the conditions $\left(H_{1}\right)-\left(H_{2}\right)$, using Young's inequality, it follows that

$$
\begin{aligned}
& E\|\zeta(x, t)\|_{L^{2}(O)}^{2}+\lambda E \int_{0}^{t}\|\zeta(x, s)\|_{H^{1}(O)}^{2} d s \\
& \quad \leq C E \int_{0}^{t}\|\zeta(x, s)\|_{L^{2}(O)}^{2} d s \quad \text { as } m \longrightarrow+\infty,
\end{aligned}
$$

where $\lambda>0$ is a constant.

Hence, our conclusion follows.

\section{The Existence of the Optimal Control}

In this section, we give our main result based on the above lemmas.

Theorem 5 (existence of the optimal control). One assumes $\left(H_{1}\right)-\left(H_{3}\right)$ hold together with the following additional conditions:

(i) the function $l\left(x, t, y^{u}(x, t), u(x, t)\right)$ is convex for each $(x, t, y, \cdot) \in Q \times R$

(ii) there exists a $u_{0}(\cdot, \cdot) \in U_{a d}$ such that the set $\{u(\cdot, \cdot) \in$ $\left.U_{a d}: J(u(\cdot, \cdot)) \leq J\left(u_{0}(\cdot, \cdot)\right)\right\}$ is bounded in $L^{2}(\Sigma)$.

Then there exists at least one optimal control for Problem (P).

Proof. Thanks to the conditions and Lemma 2, it is easy to verify that $J(u(\cdot, \cdot))$ is finite for each $u(\cdot, \cdot) \in U_{\text {ad }}$.

Now we fix a $\omega \in \Omega$, and we can find a minimizing sequence $\left\{u_{m}(\cdot, \cdot)\right\}_{m \in N} \subset U_{\text {ad }}$ for $J(u(\cdot, \cdot))$. Let $\left\{y_{m}(\cdot, \cdot)\right\}_{m \in N}$ be the corresponding states, that is, the solutions of (1) corresponding to $\left\{u_{m}(\cdot, \cdot)\right\}_{m \in N}$. By condition (ii), there exists a constant $C>0$ such that

$$
\left\|u_{m}(\cdot, \cdot)\right\|_{L^{2}(\Sigma)} \leq C, \quad \forall m \in N
$$

Hence, we can assume that

$$
u_{m}(\cdot, \cdot) \longrightarrow \bar{u}(\cdot, \cdot) \quad \text { weakly in } L^{2}(\Sigma)
$$

for some $\bar{u}(\cdot, \cdot) \in U_{\text {ad }}$ (because $U_{\text {ad }}$ is a closed set ).

Combining the conclusions of Lemmas 2 and 4, we can deduce that

$$
\begin{gathered}
E\left\|y_{m}(t)\right\|_{L^{2}(O)}^{2}+\left\|y_{m}\right\|_{L_{\mathfrak{F}}^{2}\left(\Omega \times(0, T) ; H^{1}(O)\right)}^{2} \leq C \quad \forall m \in N, \\
y_{m}(\cdot, \cdot) \longrightarrow y_{\bar{u}}(\cdot, \cdot) \quad \text { in } L^{2}\left((0, T) ; L^{2}(O)\right) \text { as } m \longrightarrow+\infty .
\end{gathered}
$$

On the other hand, by Mazur's theorem (see, for instance, [20]), we can find a sequence of convex combinations $\left\{v_{m}(\cdot, \cdot)\right\}_{m \in N}$; that is,

$$
\begin{gathered}
v_{m}(\cdot, \cdot)=\sum_{l=m}^{N(m)} \lambda_{m}^{l} u_{l}(\cdot, \cdot) \quad \text { with } \sum_{l=m}^{N(m)} \lambda_{m}^{l}=1, \\
\lambda_{m}^{l} \geq 0, \quad m \leq l \leq N(m),
\end{gathered}
$$

such that

$$
v_{m}(\cdot, \cdot) \longrightarrow \bar{u}(\cdot, \cdot) \quad \text { in } L^{2}(\Sigma) \text { as } m \longrightarrow+\infty
$$


Now, using the convexity of $l\left(x, t, y^{u}(x, t), u(x, t)\right)$ with respect to $u(x, t)$, by the dominated convergence theorem and condition $\left(\mathrm{H}_{3}\right)$, we obtain

$$
\begin{aligned}
& J(\bar{u}(\cdot, \cdot)) \\
& =E \int_{Q} L\left(x, t, y^{\bar{u}}(x, t)\right) d x d t \\
& +E \int_{\Sigma} l\left(x, t, y^{\bar{u}}(x, t), \bar{u}(x, t)\right) d \rho(x) d t \\
& +E \int_{O} r\left(x, y^{\bar{u}}(x, T)\right) d x \\
& =\lim _{m \rightarrow+\infty} E \int_{\Sigma} l\left(x, t, y^{\bar{u}}(x, t), v_{m}(x, t)\right) d \rho(x) d t \\
& +E \int_{Q} L\left(x, t, y^{\bar{u}}(x, t)\right) d x d t+E \int_{O} r\left(x, y^{\bar{u}}(x, T)\right) d x \\
& =\lim _{m \rightarrow+\infty} \sum_{l=m}^{N(m)} \lambda_{m}^{l} \\
& \times\left(J\left(u_{l}(x, t)\right)\right. \\
& +E \int_{\Sigma}\left(l\left(x, t, y^{\bar{u}}(x, t), u_{l}(x, t)\right)\right. \\
& \left.-l\left(x, t, y^{u_{l}}(x, t), u_{l}(x, t)\right)\right) d \rho(x) d t \\
& +E \int_{Q}\left(L\left(x, t, y^{\bar{u}}(x, t)\right)-L\left(x, t, y^{u_{l}}(x, t)\right)\right) d x d t \\
& \left.+E \int_{O}\left(r\left(x, y^{\bar{u}}(x, T)\right)-r\left(x, y^{u_{l}}(x, T)\right)\right) d x\right) \\
& \leq \inf _{u(\cdot,) \in U_{\mathrm{ad}}} J(u(\cdot, \cdot)) \\
& +\lim _{m \rightarrow+\infty} \sum_{l=m}^{N(m)}\left(E \int_{Q} M\left|y_{u_{l}}-y_{\bar{u}}\right| d x d t\right. \\
& +E \int_{\mathrm{Q}} M\left|y_{u_{l}}-y_{\bar{u}}\right| d x d t \\
& \left.+E \int_{O} M\left|y_{u_{l}}-y_{\bar{u}}\right| d x\right) \\
& \leq \inf _{u(\cdot,) \in U_{\mathrm{ad}}} J(u(\cdot, \cdot))+C \lim _{m \rightarrow+\infty} \sum_{l=m}^{N(m)}\left\|y_{u_{l}}-y_{\bar{u}}\right\|_{L^{2}\left((0, T) ; L^{2}(O)\right)} \\
& =\inf _{u(\cdot,) \in U_{\mathrm{ad}}} J(u(\cdot, \cdot)) .
\end{aligned}
$$

That means $\bar{u}(\cdot, \cdot)$ is an optimal control for Problem (P).

Remark 6. In this paper, we give a new method to study the existence of the optimal control for stochastic control problems. And we also can see that, compared to the necessary conditions, we need to give some additional conditions to obtain the existence result. So the existence problems are harder to be considered.

\section{Conflict of Interests}

The authors declare that there is no conflict of interests regarding the publication of this paper.

\section{Acknowledgments}

This work was partially supported by the Special Fund of Basic Scientific Research of Central Colleges (Grant no. CZQ14021). This work was partially supported by the Teaching Research Fund of South-Central University for Nationalities (JYX13023).

\section{References}

[1] L. S. Pontryagin, V. G. Boltyanskti, R. V. Gamkrelidze, and E. F. Mischenko, The Mathematical Theory of Optimal Control Processes, Interscience, John Wiley \& Sons, New York, NY, USA, 1962.

[2] J. L. Lions, Optimal Control of Systems Governed by Partial Differential Equations, Springer, 1971.

[3] A. Bensoussan, "Stochastic maximum principle for distributed parameter systems," Journal of the Franklin Institute, vol. 315, no. 5-6, pp. 387-406, 1983.

[4] D. P. Bertsekas, Dynamic Programming and Optimal Control, Athena Scientific, Belmont, Mass, USA, 2nd edition, 2000.

[5] U. G. Haussmann, "General necessary conditions for optimal control of stochastic systems," Mathematical Programming Study, no. 6, pp. 30-48, 1976.

[6] H. J. Kappen, "An introduction to stochastic control theory, path integrals and reinforcement learning," in Proceedings of the 9th Granada Seminar on Computational Physics, pp. 149-181, American Institute of Physics, 2007.

[7] J. Yong and X. Y. Zhou, Stochastic Controls: Hamiltonian Systems and HJB Equations, vol. 43 of Applications of Mathematics, Springer, New York, NY, USA, 1999.

[8] X. Y. Zhou, "On the necessary conditions of optimal controls for stochastic partial differential equations," SIAM Journal on Control and Optimization, vol. 31, no. 6, pp. 1462-1478, 1993.

[9] I. Lasiecka and R. Triggiani, "Exact controllability of the wave equation with Neumann boundary control," Applied Mathematics and Optimization, vol. 19, no. 3, pp. 243-290, 1989.

[10] T. T. Li, "Exact controllability for quasilinear hyperbolic systems and its application to unsteady flows in a network of open canals," Mathematical Methods in the Applied Sciences, vol. 27, no. 9, pp. 1089-1114, 2004.

[11] E. Casas, "Pontryagin's principle for state-constrained boundary control problems of semilinear parabolic equations," SIAM Journal on Control and Optimization, vol. 35, no. 4, pp. 12971327, 1997.

[12] S. Chai, "Stabilization of thermoelastic plates with variable coefficients and dynamical boundary control," Indian Journal of Pure and Applied Mathematics, vol. 36, no. 5, pp. 227-249, 2005.

[13] R. Buckdahn, B. Labed, C. Rainer, and L. Tamer, "Existence of an optimal control for stochastic control systems with nonlinear cost functional," Stochastics, vol. 82, no. 3, pp. 241-256, 2010. 
[14] M. H. A. Davis, "On the existence of optimal policies in stochastic control," SIAM Journal on Control and Optimization, vol. 11, pp. 587-594, 1973.

[15] E. Karoui Nicole, D. Nguyen, and J.-P. Monique, "Compactification methods in the control of degenerate diffusions: existence of an optimal control," Stochastics, vol. 20, no. 3, pp. 169-219, 1987.

[16] C. B. Wan and M. H. A. Davis, "Existence of optimal controls for stochastic jump processes," SIAM Journal on Control and Optimization, vol. 17, no. 4, pp. 511-524, 1979.

[17] E. Casas, L. A. Fernández, and J. M. Yong, "Optimal control of quasilinear parabolic equations," Proceedings of the Royal Society of Edinburgh A: Mathematics, vol. 125, no. 3, pp. 545-565, 1995.

[18] T. I. Seidman and H. Zhou, "Existence and uniqueness of optimal controls for a quasilinear parabolic equation," The SIAM Journal on Control and Optimization, vol. 20, no. 6, pp. 747-762, 1982.

[19] H. Yu and B. Liu, "Optimality conditions for stochastic boundary control problems governed by semilinear parabolic equations," Journal of Mathematical Analysis and Applications, vol. 395, no. 2, pp. 654-672, 2012.

[20] I. Ekeland and R. Temam, Analyse Convexe et Problèmes Variationnels, Dunod, Paris, Farnce, 1974. 


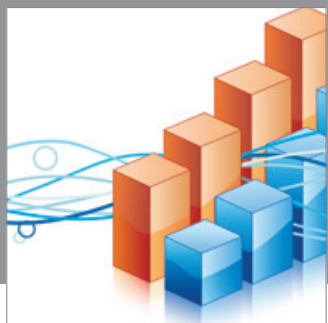

Advances in

Operations Research

mansans

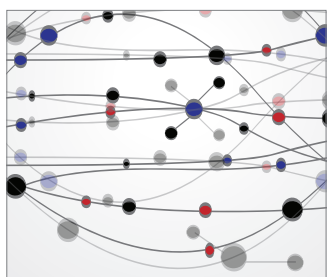

The Scientific World Journal
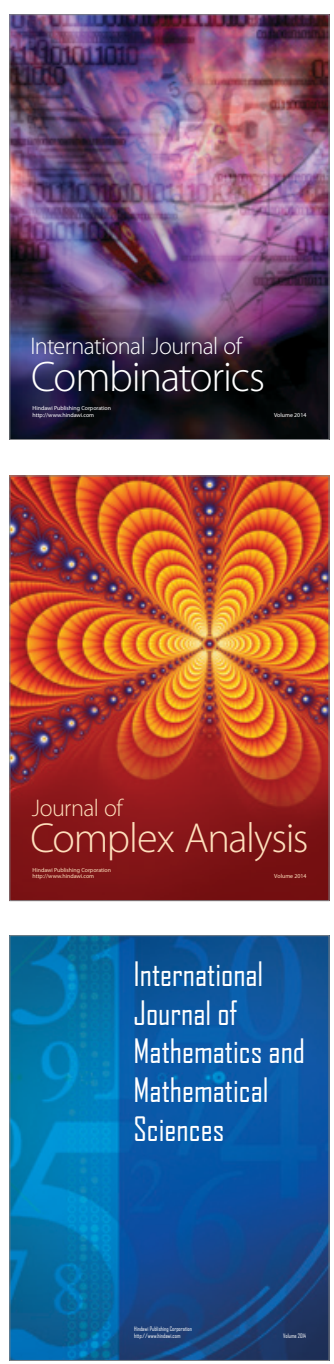
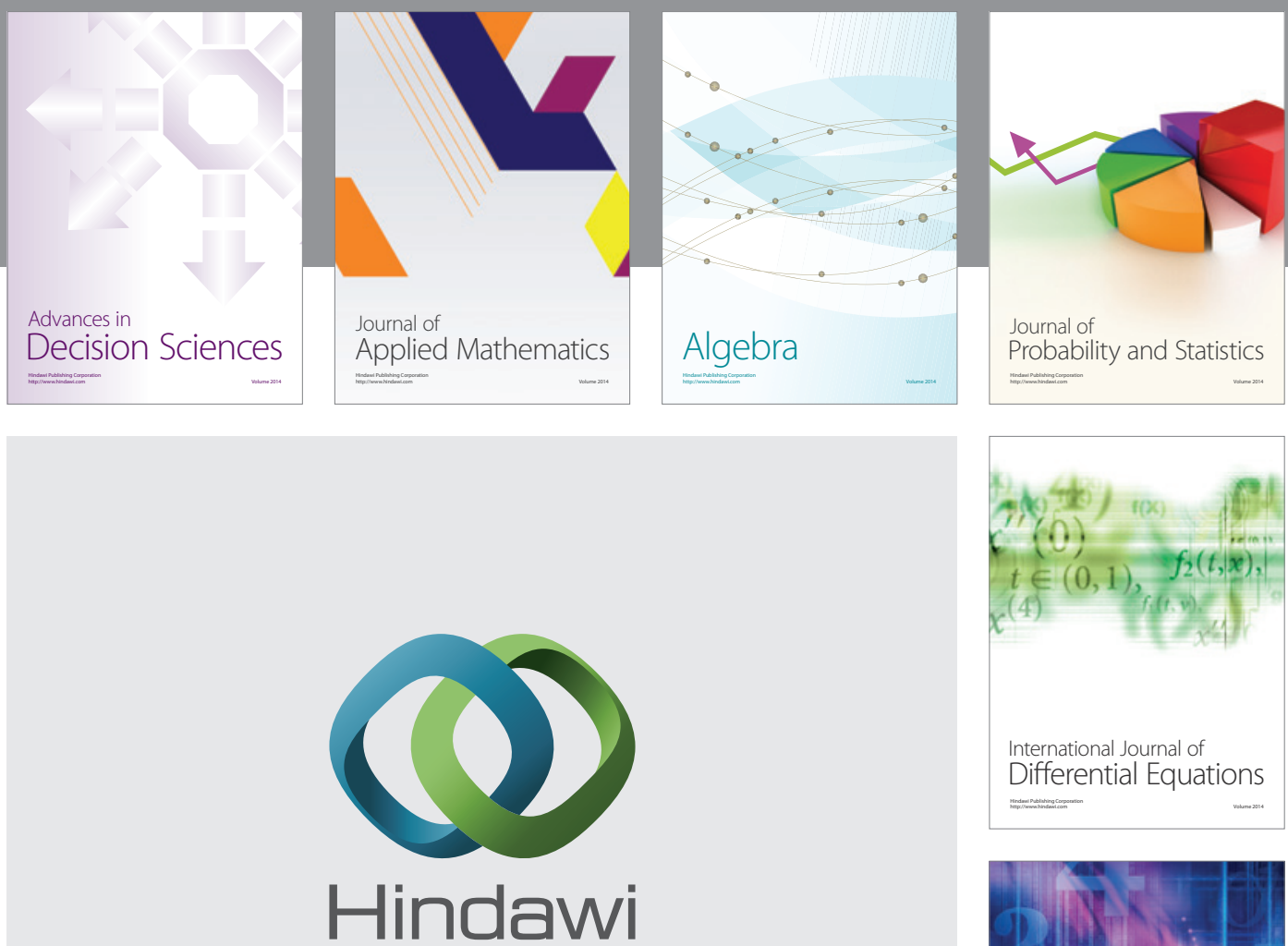

Submit your manuscripts at http://www.hindawi.com
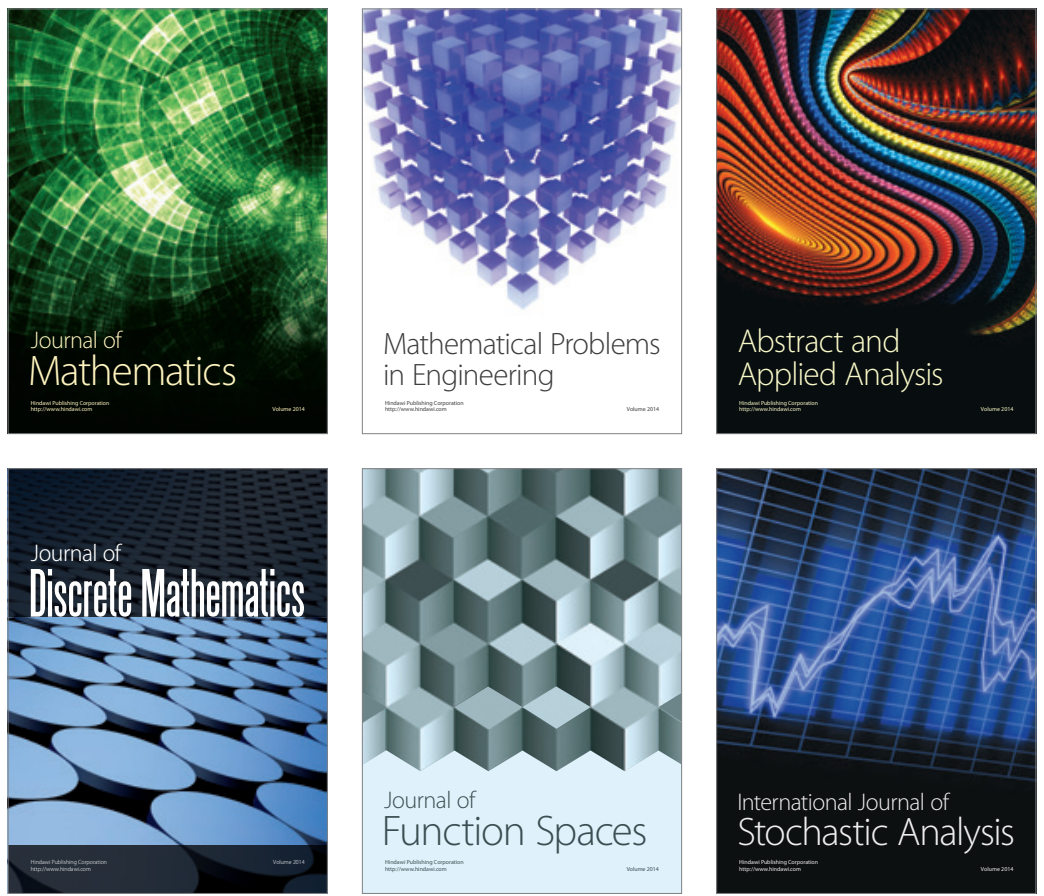

Journal of

Function Spaces

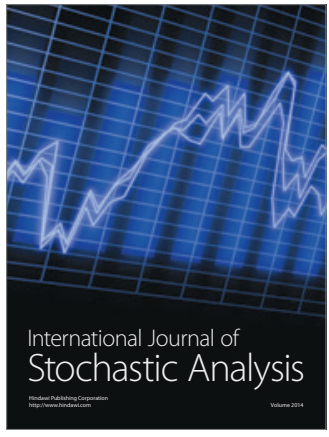

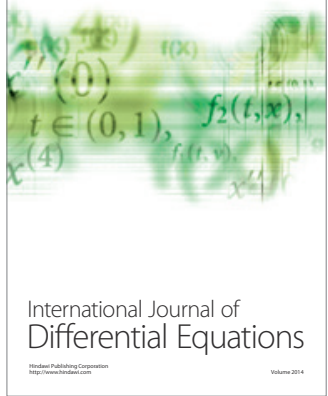
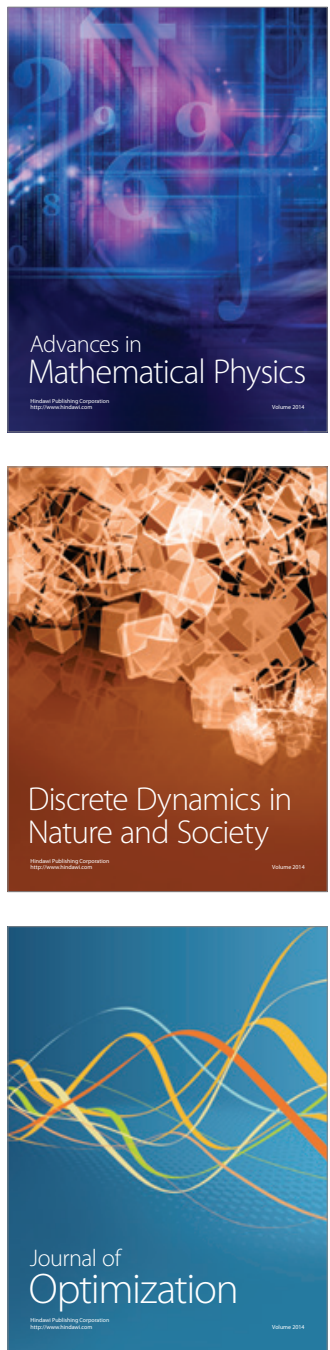